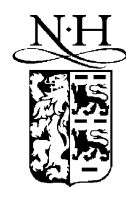

ELSEVIER

\title{
Stochastic optimization for a tip-tilt adaptive correcting system ${ }^{\star 2}$
}

\author{
M.S. Zakynthinaki ${ }^{\mathrm{a}, *}$, Y.G. Saridakis ${ }^{\mathrm{b}}$ \\ a Departament de Matemática Aplicada I, Universitat Politécnica de Catalunya, Diagonal 647, E-08028 Barcelona, Spain \\ ${ }^{\mathrm{b}}$ Applied Mathematics and Computer Laboratory, Department of Sciences, Technical University of Crete, 73100, Chania, Greece
}

Received 24 April 2002; received in revised form 26 June 2002; accepted 13 July 2002

\begin{abstract}
We present computer simulations of a tip-tilt adaptive optics system, where stochastic optimization is applied to the problem of dynamic compensation of atmospheric turbulence. The system uses a simple measure of the light intensity that passes through a mask and is recorded on the image plane, to generate signals for the tip-tilt mirror. A feedback system rotates the mirror adaptively and in phase with the rapidly changing atmospheric conditions. Computer simulations and a series of numerical experiments investigate the implementation of the method in the presence of drifting atmosphere. In particular, the study examines the system's sensitivity to the rate of change of the atmospheric conditions and investigates the optimal size of
\end{abstract} the mirror's masking area and the algorithm's optimal degree of stochasticity.

() 2002 Elsevier Science B.V. All rights reserved.

PACS: 02.60.Pn; 07.05.Tp; 95.75.Qr; 95.75Pq

Keywords: Stochastic optimization; ALOPEX algorithm; Adaptive wavefront correction; Tip-tilt mirror; Masking

\section{Introduction}

Atmospheric layers act, due to turbulence, as a distorting optical system which causes parallel light rays to diverge. A typical short exposure image (exposure time $<2-20 \mathrm{msec}$ ), formed by viewing a point source through turbulence, does not consist of a single diffraction pattern with a diameter fixed by the diffraction limit of the telescope, but rather of a number of su-

\footnotetext{
This work was supported by the Greek General Secretariat of Research and Technology under the grant PENED-107.527.

* Corresponding author. Partially supported by the grant SB 2000-0396 from the Secretaría de Educación y Universidades and Fondo Social Europes.

E-mail addresses: marzak@mailcity.com (M.S. Zakynthinaki), yiannis@science.tuc.gr (Y.G. Saridakis).
}

perimpozed speckles, microscopic images of the point source, which are distributed over a diameter determined by the severity of turbulence (see Section 2.1). This phenomenon is referred to as atmospheric seeing [1]. Due to seeing, the capabilities of even the larger ground based telescopes remain unexploited.

Today it is technologically possible to smooth out the distorting effects of the earth's atmosphere by the use of adaptive optics systems, which are able to adaptively cancel out, or at least minimize, atmospheric seeing. The basic idea of adaptive optics was formulated in 1953 by Babcock [2,3]. However, adaptive optics systems were developed only recently, due to the technological limitations of the implementation of such systems. The basic limitation of an adaptive optics system lies in the fact that a large number of highly 
expensive and complex elements are needed for the wavefront sensing. By use of such sensors, the distorted wavefront is repeatedly evaluated and continuously corrected, in real-time, through a rotating and/or a deformable mirror. In this way the spatial resolution of the image of a star can be effectively improved up to the theoretical diffraction limit of the telescope system under use. Adaptive optics systems are currently in use in the world's largest astronomical observatories.

The present study investigates the development of an alternative adaptive optics method based on stochastic optimization, in which the expensive wavefront sensors will be replaced by a simple and cost effective system. According to Noll [4], the so-called tip-tilt distortion accounts for $85 \%$ of the aberration induced upon a wavefront, which is passing though a turbulent atmosphere. The tip-tilt is referred to as the first order distortions which define, in the case of atmospheric seeing, the time varying wavefront gradients. Assuming that the centroid of an image is the centre of its intensity distribution, the tip-tilt distortion results in a displacement of the centroid and hence a blurring of the image. The correcting element for the system under study is assumed to be a flat mirror, which can be tilted along two orthogonal planes to correct the motion of the image. For the higher orderbut less significant—corrections, a deformable mirror $[1,5]$ is necessary. However, the primary concern of any adaptive optics system is the first order tip-tilt correction, by which a significant part of the distortions caused by seeing may be eliminated. Tip-tilt correction may also account for the minimization of any thermal effects within the telescope enclosure and above the surface of the mirror. In addition, any optical aberrations caused by micro-movements or other possible external factors can be smoothed out.

In the simulated tip-tilt adaptive optics system presented here, the wavefront sensor is to be replaced by a mask applied directly to the image, on the image plane. The mask allows only a fraction of the light information to pass through its narrow aperture. Behind the mask there is a light detector, evaluating the total light intensity passing through the mask and being recorded on the central area of the image plane. This central image area behind the mask's aperture will be referred to as the masking area. A computer, by means of the optimization algorithm, drives the tip-tilt mirror in such a way as to bring the centroid (i.e. the centre of the light intensity of the image) over the aperture of the mask. By means of this optimization method, the restoration of the centroid of the image is achieved, in real time, resulting in the improvement of the light distribution of the long exposure image recorded (exposure time $>2-20 \mathrm{msec}$ ). The process is repeated at each time step. Fig. 1 illustrates the optimization process.

For the simulation purposes, atmospheric turbulence is modeled following the principles of the relative Kolmogorov theory [6] and by using a sequence of phase screens. A phase screen is a two-dimensional distribution of phase fluctuations, introduced into the optical system to simulate the effects of the turbulent atmosphere. These phase fluctuations, are numerically generated $[1,5,7]$ by the so-called random mid-point displacement algorithm, used for the same purposes by Lane et al. [7] (see Section 2.2). The phase fluctuations are transported across the telescope aperture according to local wind conditions.

For the optimization of the image, ALOPEX stochastic optimization is used (see Section 3 and [1,5, 8-11]). ALOPEX optimization methods are driven by the parallel incoherent dithering of the control variables and the time dependence of the feedback. Their main advantage is that no knowledge of the dynamics of the system is required [8,9]. The method's effectiveness and practicality depend on the ability to follow the motion of the turbulent wavefronts. ALOPEX stochastic optimization algorithm is chosen due to its speed of convergence and its simple implementation $[1,5,10,11]$.

In the present study we apply the ALOPEX stochastic optimization method to the problem of real time sharpness restoration of the image of a point star. We investigate

- the typical method's behaviour during the process of image restoration (Section 4.2);

- the optimal size of the masking area (Section 4.3);

- the system's sensitivity to the rate of change of atmospheric distortions (Section 4.4.1);

- the relation of the algorithm's optimal mean noise amplitude and the rate of change of atmospheric distortions, in the case of bad weather conditions (Section 4.4.2). 


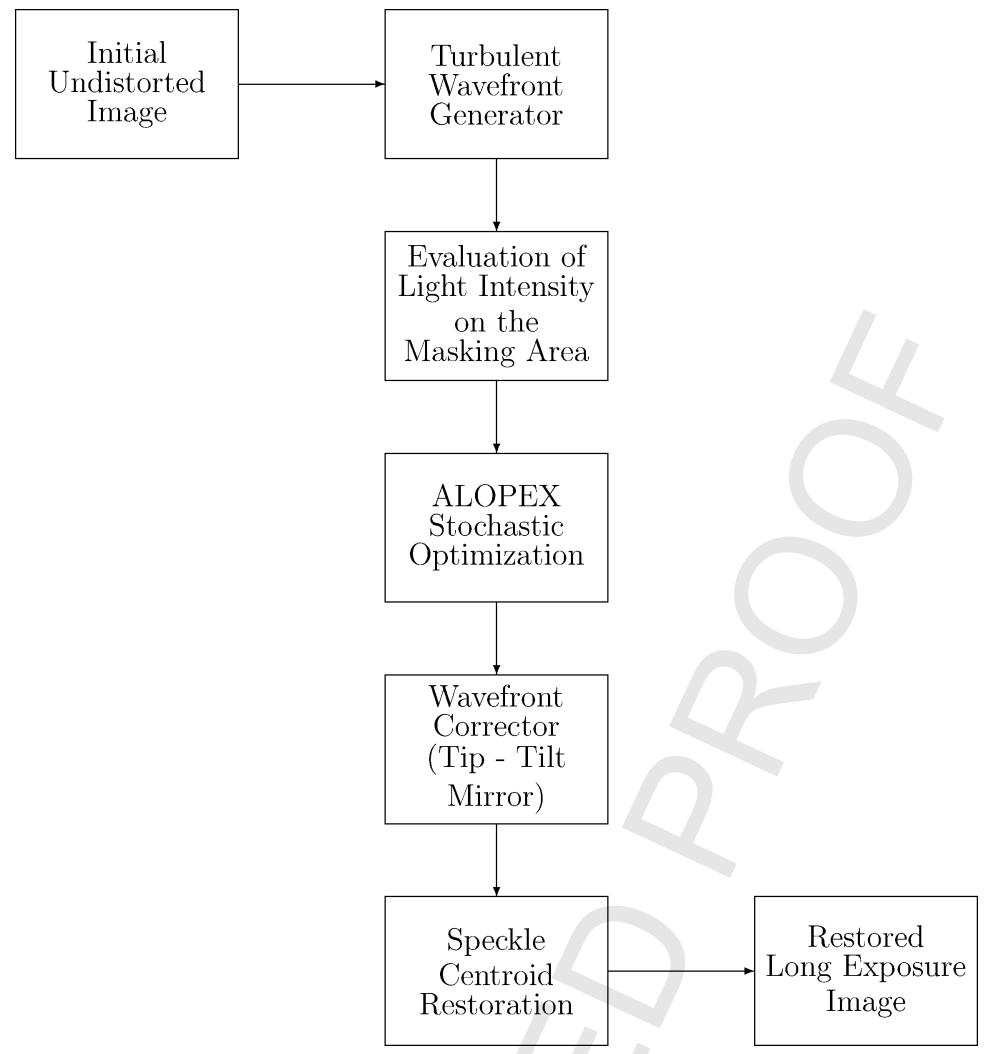

Fig. 1. A time step of the simulated adaptive optics system.

\section{Computer simulations and background}

\subsection{Atmospheric distortion and adaptive correction}

Let us assume a point source at infinity. Coherent light rays coming from the point source and passing through the turbulent atmospheric layers, deviate from planarity. The distorted information passes through the circular telescope aperture $(\rho, \theta)$ and forms an image of the point star on the image plane $(r, \psi)$. The light distribution of any point on the image plane, in the presence of interfering atmospheric distortions, can be given by the following modified Fresnel-Kirchhoff integral [1,5,12],

$I(r, \psi)=\left|\int_{0}^{1} \int_{0}^{2 \pi} \mathrm{e}^{\mathrm{i} k[\delta(\rho, \theta)-r \rho \cos (\theta-\psi)]} \rho \mathrm{d} \theta \mathrm{d} \rho\right|^{2}$,

where $k$ is the wave number of the incident wave.
The real function $\delta(\rho, \theta)$ in Eq. (1) includes the optical effects of both the distorting atmosphere $\phi(\rho, \theta)$ and the correcting mirror $\phi_{c}(\rho, \theta)$. In the absence of any distortions $\delta(\rho, \theta)=0$, while in general

$\delta(\rho, \theta)=\phi(\rho, \theta)-\phi_{c}(\rho, \theta)$.

Function $\delta(\rho, \theta)$ is of great interest in adaptive optics. However, it is not directly measurable, as far as optical wavelengths are concerned. The use of an alternative method for the estimation of the image quality and the corrections needed would therefore speed up the process of image optimization in any adaptive optics system.

In the adaptive optics system presented here, the problem of minimization of $\delta(\rho, \theta)$ (maximum possible elimination of atmospheric distortions) is solved via the maximization of the total light intensity passing through the mask. As a result of keeping the light intensity inside the masking area at maximum levels, less spreading of the light distribution is achieved. As- 
suming a central masking area of radius $R$, the total light intensity inside this area can be represented by the integral

$$
S_{\text {tip tilt }} \equiv \int_{-R}^{R} \int_{0}^{2 \pi} I(r, \psi) r \mathrm{~d} \psi \mathrm{d} r,
$$

which can be used to evaluate the cost function $S_{\text {tip tilt }}$ that describes the simulated system.

For a tip-tilt system, i.e. a system that only corrects the two lowest sources of aberrations, the correcting phase $\phi_{c}(\rho, \theta)$ is a function of the slope of the mirror's surface and is for simplicity expressed in Cartesian coordinates as

$\phi_{c}(x, y)=a_{1} x+a_{2} y$.

The variables $x$ and $y$ represent the phase gradients in the $x$ - and $y$-direction, while the tip and tilt components $a_{1}, a_{2}$ are variables determining the slope of the mirror. Changes in the tip and tilt components lead to a movement of the centroid of the star's image.

Fig. 2 shows the image of a point star, generated by means of Eq. (1), under the assumption that no atmospheric distortion is present. Such an image consists of a number of diffraction rings around a central light disk referred to as the airy disk of the image. Theoretically the angular width of the airy disk can be calculated using the relation

$\delta \theta \approx 1.22 \frac{\lambda}{D} \approx 0.25 \frac{\lambda(\mu \mathrm{m})}{D(\mathrm{~m})} \operatorname{arcsec}$,

where $\lambda$ and $D$ denote the wavelength of the incident light and the diameter of the telescope aperture, respectively. Obviously the quantity $\delta \theta$ gives a theoretical limit of the resolution of a telescope of a given diameter $D$, since two neighboring sources are distinguishable only when placed at a minimum distance $\delta \theta$ apart. In our simulation, the light was considered monochromatic with a wavelength of $\lambda=0.5 \mu \mathrm{m}$.

The spatial resolution of ground based telescopes with diameter greater than $D>30 \mathrm{~cm}$ is, however, considerably less than the diffraction limit given by Eq. (5), even under good weather conditions. For visible wavefronts the light distribution typically appears spread on an area 10-20 times larger than the one which is theoretically expected. If, for given seeing,

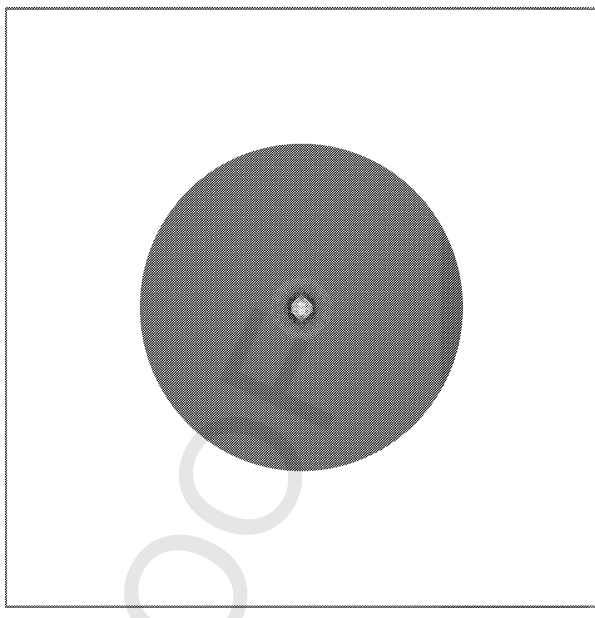

Fig. 2. The image of a point star-no disturbing atmosphere is present, (a) as shown on the image plane and (b) irradiance distribution.

the angular resolution of a star is $\delta \theta$, then Eq. (5) can be re-written in the form

$\delta \theta \approx 1.22 \frac{\lambda}{r_{0}}$

where $r_{0}$ is the Fried's coherence length of atmospheric turbulence $[1,13]$. For given $\lambda$, the parameter $r_{0}$ experimentally represents the maximum telescope diameter that leaves light information unaffected by turbulence. Theoretically $r_{0}$ gives the characteristic length above which turbulent atmospheric movement remain statistically uncorrelated. The value of $r_{0}$ is time-varying 5 6 (

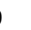

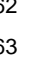
64 . .

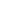
. , 4 75 . , 


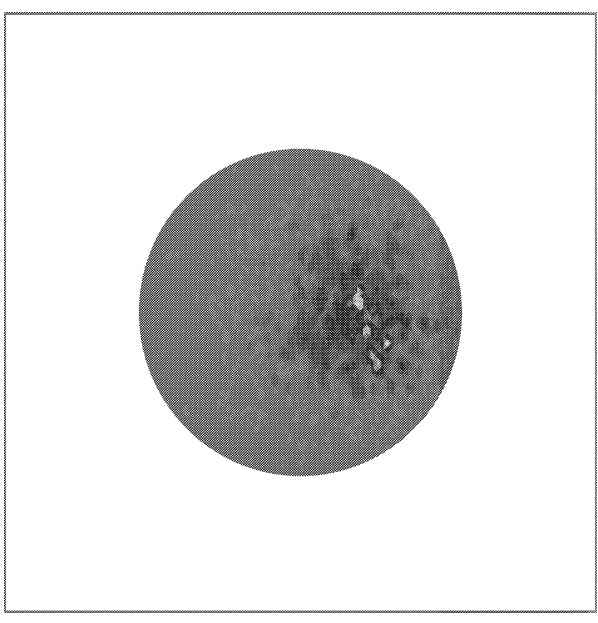

(a)

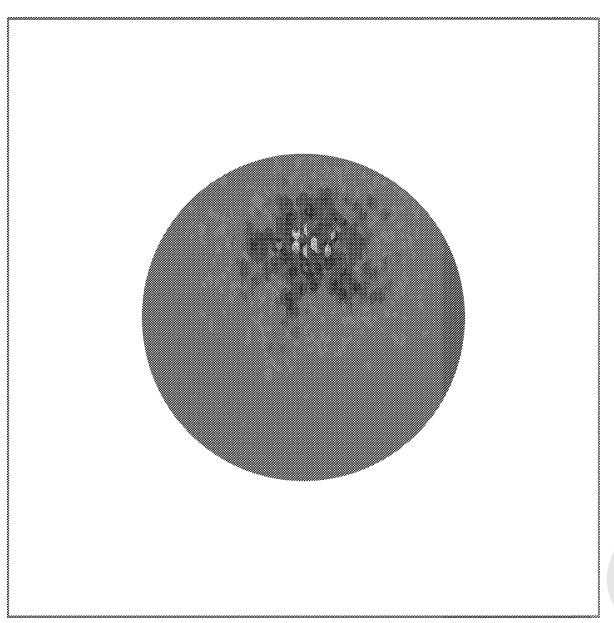

(c)

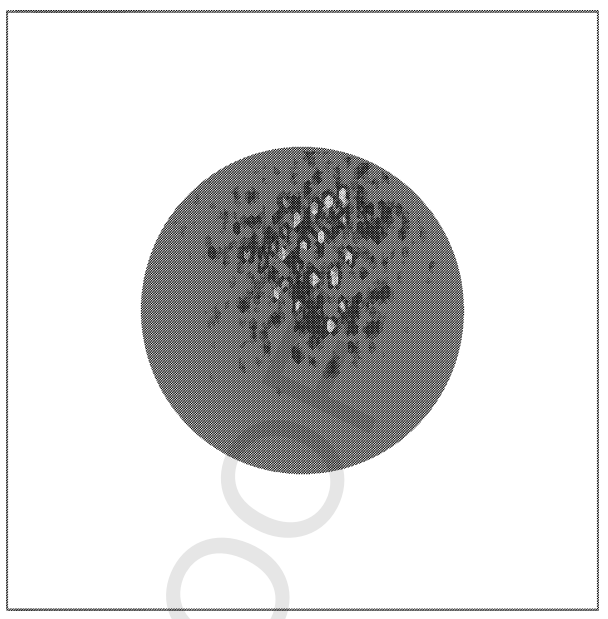

(b)

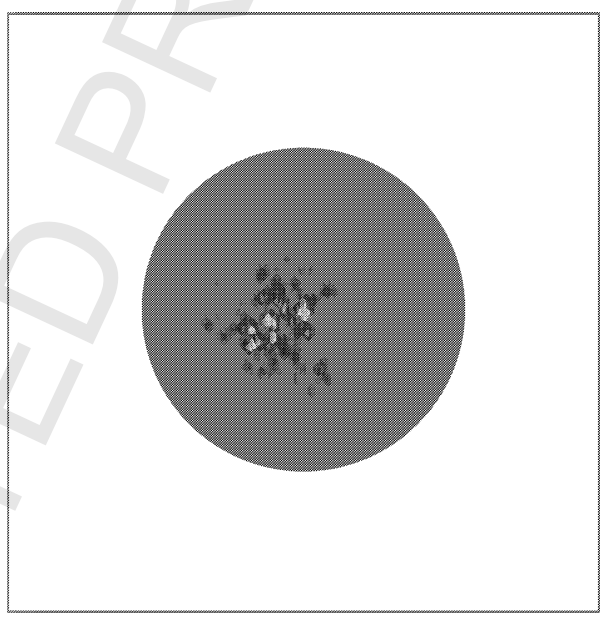

(d)
Fig. 3. Speckle samples. and depends on the severity of turbulence and the wavelength used.

Evidently a typical telescope diameter $D$ is larger than the coherence length $r_{0}$. As a result, the telescope pupil can be thought of as a dense collection of $K$ microscopic lenses of diameter $r_{0}$, which densely cover the pupil plane. This number $K$ can be approximated by

$K \approx\left(\frac{D}{r_{0}}\right)^{2}$.

The $K$ microscopic tilted lenses result in a random interference of light rays and the formation of $K$ micro- scopic images on the image plane. Each microscopic image of the star is of diameter $\lambda / D$ approximately, while the displacement of each image from the central image area is random. These microscopic images of the point source are referred to as speckles (see Fig. 3). The speckle pattern moves as a whole on the image plane. Due to its random motion and changes in shape, the time integrated, long exposure image, appears spread on a large area on the image plane. It is worth noticing that the speckles observed in the images of Fig. 3 are the result of bad atmospheric seeing as, for the particular simulation, there was $D / r_{0}=$ 17.3.

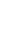

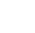

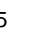

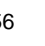

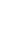
9 (1)

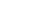

(1)
5 6 7

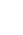
(1) 2 74 75 76 8 9 
The Strehl Ratio $(S R)$ is defined as a restoration criterion, used to estimate the sharpness of the restored images,

$$
S R=\frac{I(0)}{M(0)},
$$

with $M(r)$ being the irradiance distribution of the undistorted image. The value of $S R$ gives a measure of the central light intensity of a restored image, in comparison to the central intensity peak of the optimal undistorted image (see Fig. 2). Evidently the maximum value of the Strehl Ratio is $S R=1$. For the simulation of the point star (see Fig. 2) we normalized the peak of the irradiance distribution to unity. Thus, for an undistorted light distribution $M(r)$, there is $S R=M(0)=1$. Under this assumption, the value of the $S R$ of any undistorted image can be calculated by the relation

$S R=I(0)$

(see Eq. (7)).

\subsection{Atmospheric simulation}

The atmospherically distorted wavefront at the telescope pupil is modeled in terms of Kolmogorov theory [6], which predicts the statistical properties of the fluctuations of the refractive index and leads to equations which describe the turbulence induced by the thin layers within the atmosphere. For the simulation of the atmospheric distortion, a phase screen, i.e. a two-dimensional distribution of phase fluctuations satisfying a Kolmogorov spectrum, is introduced to the optical system at each time step of the optimizing algorithm (recall Fig. 1).

Lane, Glindemann and Dainty [7] used the random mid-point displacement method, previously applied to the computer generation of artificial landscapes, for a Kolmogorov turbulent screen generation. The same technique is applied in the present study as well (see also [1,5]), for the generation of a $65 \times 65$ Kolmogorov sampling grid. The algorithm is based on making a coarsely sampled approximation to the turbulent atmosphere and the subsequent refinement of the successive smaller localized regions. For more details see $[1,5,7]$.

Let us assume the $n$th time step of the optimization process. The numerical generation of the time evolving turbulent phase screen $\phi(\rho, \theta)^{(n)}$ begins with four independent starting samples $\alpha^{(n)}, \beta^{(n)}, \gamma^{(n)}$ and $\delta^{(n)}$, generated by a combination of the Gaussian variables $R^{c(n)}$ and $R^{d(n)}$, of variance $\sigma_{c}$ and $\sigma_{d}$, respectively [7]:

$\alpha^{(n)}=R_{\alpha}^{c(n)}+0.5 R_{\alpha \delta}^{d(n)}$,

$\beta^{(n)}=R_{\beta}^{c(n)}+0.5 R_{\beta \gamma}^{d(n)}$,

$\gamma^{(n)}=R_{\gamma}^{c(n)}-0.5 R_{\beta \gamma}^{d(n)}$,

$\delta^{(n)}=R_{\delta}^{c(n)}-0.5 R_{\alpha \delta}^{d(n)}$

with $\sigma_{c}$ and $\sigma_{d}$ satisfying the relations

$2 \sigma_{c}^{2}+\frac{\sigma_{d}^{2}}{2}=6.88\left(D / r_{0}\right)^{5 / 3}$,

$2 \sigma_{c}^{2}+\sigma_{d}^{2}=6.88\left(\sqrt{2} D / r_{0}\right)^{5 / 3}$.

The time dependence of the Gaussian variables $R^{c(n)}$ and $R^{d(n)}$ which provide the four starting samples $\alpha^{(n)}, \beta^{(n)}, \gamma^{(n)}$ and $\delta^{(n)}$ of the turbulent phase screen, as described by Eqs. (8), is directly related to local atmospheric conditions [1]: Assume the number $x^{(n)}$ which moves across the interval $[0,1]$ at a rate which depends upon the rate of change of the atmospheric conditions. The Gaussian variables $R_{j}^{i(n)}$, where $i=c, d$ and $j=\alpha, \beta, \gamma, \delta, \alpha \delta, \beta \gamma$, as in Eqs. (8), are then calculated by the relation

$R_{j}^{i(n)}= \pm \sigma_{i} \sqrt{-2.0 \cdot \log \left(x^{(n)}\right)}$.

For a more detailed description of the simulation of the time evolving phase screens, see [1].

Having calculated the four starting samples $\alpha^{(n)}$, $\beta^{(n)}, \gamma^{(n)}$ and $\delta^{(n)}$, a new point $m^{(n)}$ can be generated by linear interpolation and the addition of a displacement $\epsilon^{(n)}$ :

$m^{(n)}=\frac{\alpha^{(n)}+\beta^{(n)}+\gamma^{(n)}+\delta^{(n)}}{4}+\epsilon^{(n)}$.

If $h^{(n)}$ is defined to be the distance between two adjacent samples on the sampling grid, the variance of the displacement $\epsilon^{(n)}$ can be required [7] to be equal to $\sigma_{\epsilon}^{2}=0.6091 h^{5 / 3}$. It is worth noticing that, as the sampling grid becomes finer, the variance of the displacement is also reduced. The procedure continues by another linear interpolation between the corner points and again by the addition of displacements.

As in [7], the calculation of a new point by use of a point at the edge of the grid requires the addition of 


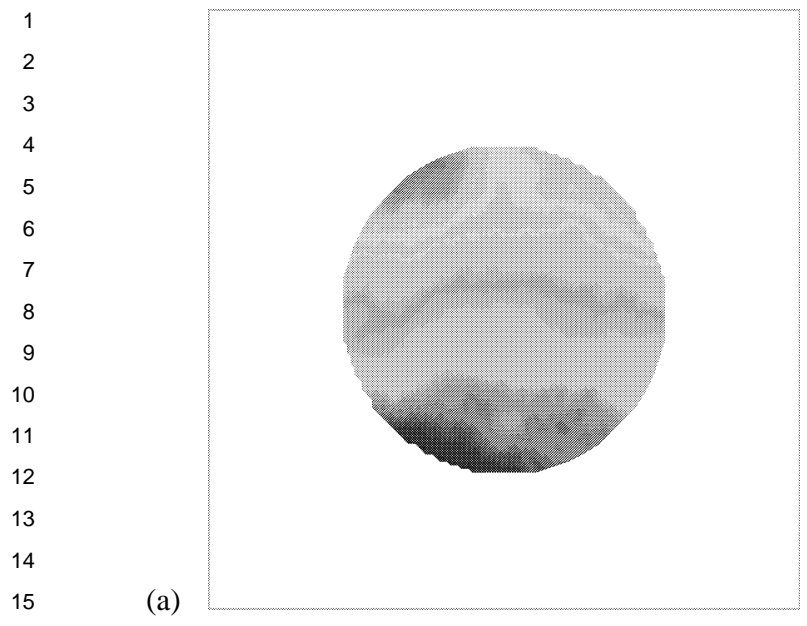

(b)
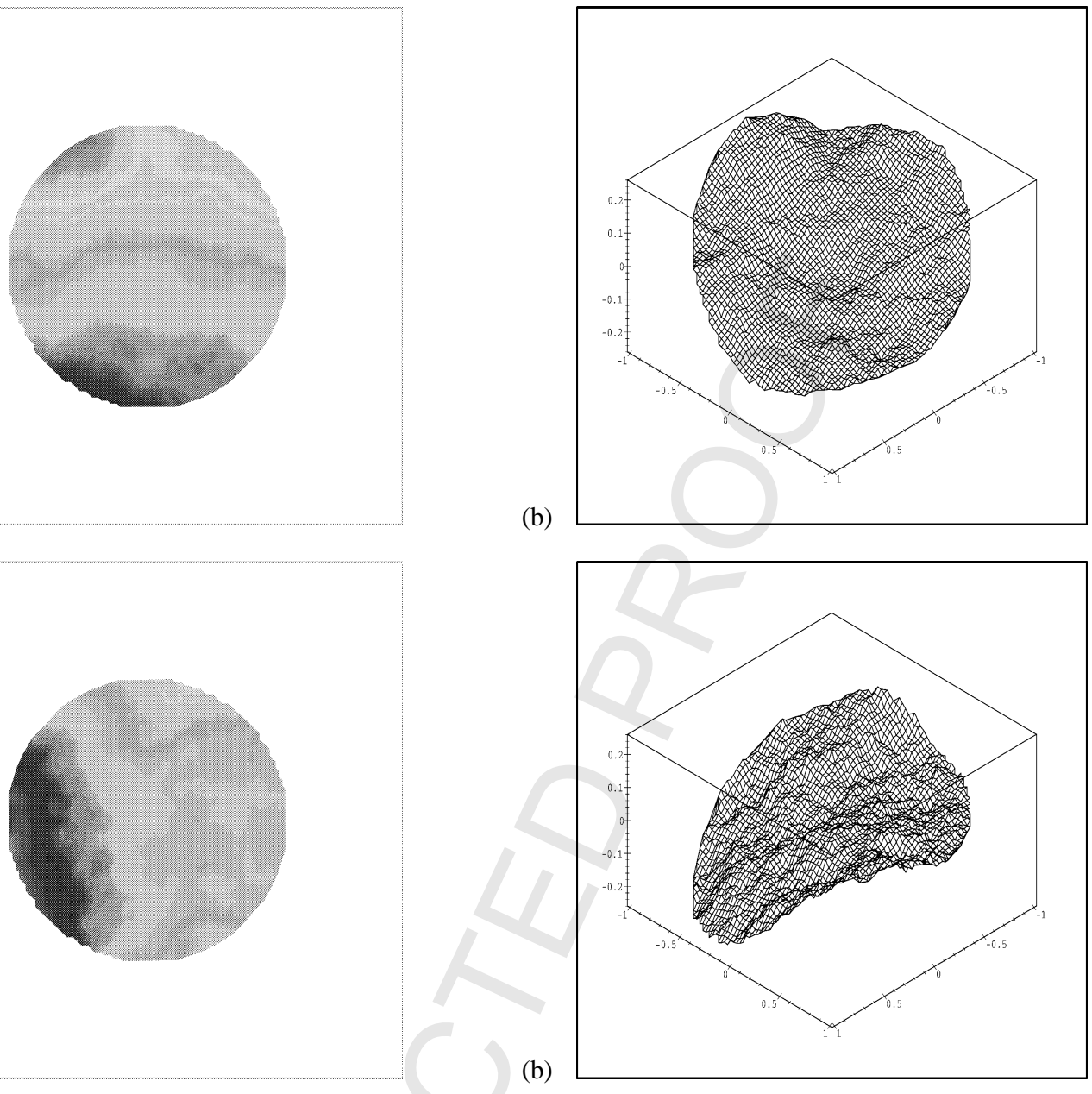

(b)

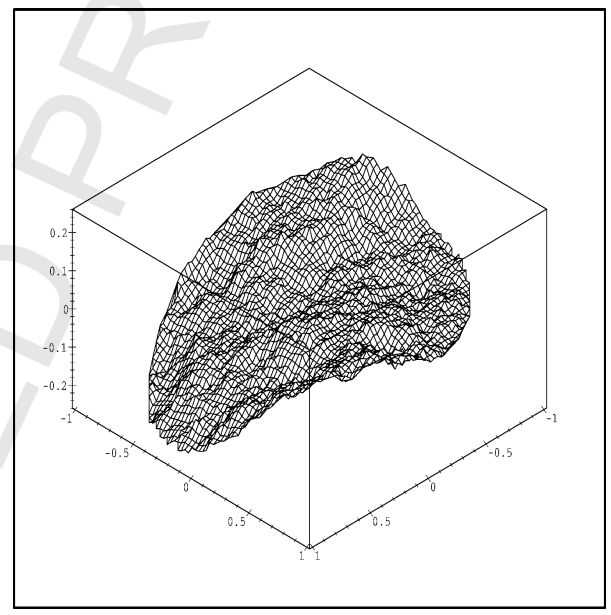

(b)

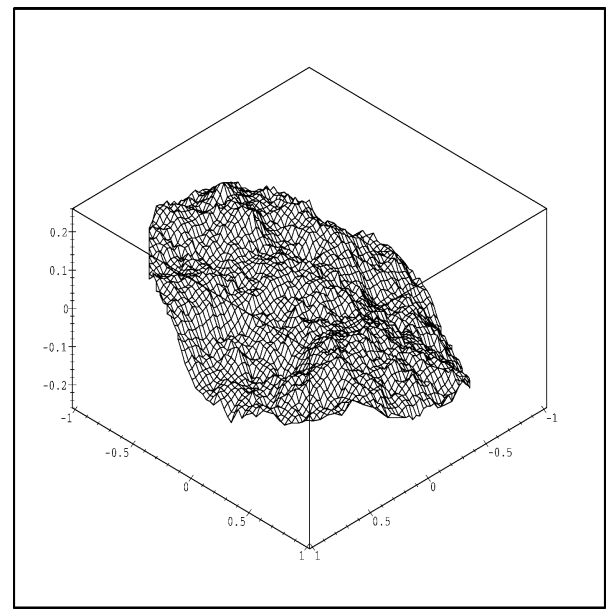

Fig. 4. Samples of turbulent phase screens (a) two-dimensional projection, (b) functional form. 
a displacement $\eta^{(n)}$ with variance $\sigma_{\eta}^{2}=0.4471 h^{5 / 3}$. On the left edge, for example, the new point $m_{\alpha \beta}^{(n)}$ is formed according to the rule $m_{\alpha \beta}^{(n)}=\frac{\alpha^{(n)}+\beta^{(n)}}{2}+\eta^{(n)}$. For a more detailed description and a discussion of this interpolation/displacement procedure see [1,5,7].

Fig. 4 shows three samples of simulated turbulent screens. For the purposes of the present study, phase screens simulating turbulence were calculated for a wide range of atmospheric conditions, starting from $D / r_{0}=3.25$ to $D / r_{0}=43.3$.

\section{ALOPEX stochastic optimization}

For the purpose of optimization, let $f \equiv f\left(x_{1}, x_{2}\right.$, $\left.\ldots, x_{N} ; y_{1}, y_{2}, \ldots, y_{M}\right)$ denote the cost function, that is the function that describes the system. Function $f$ depends on a set of $N$ parameters $x_{1}, x_{2}, \ldots, x_{N}$, called the control variables, and a set of $M$ parameters $y_{1}, y_{2}, \ldots, y_{M}$, that are not under control, and may be internal or external parameters of the system. As the set of the $M$ parameters $y_{1}, y_{2}, \ldots, y_{M}$ are not under control, we can re-write the cost function $f$ using the control variables $x_{1}, x_{2}, \ldots, x_{N}$ only,

$$
\begin{aligned}
f & \equiv f\left(x_{1}, x_{2}, \ldots, x_{N} ; y_{1}, y_{2}, \ldots, y_{M}\right) \\
& \equiv f\left(x_{1}, x_{2}, \ldots, x_{N}\right) .
\end{aligned}
$$

The stochastic optimization algorithm used for the maximization of the cost function is the socalled ALOPEX (ALgorithm Of Pattern EXtraction) algorithm. It was originally devised in $[8,9]$ for the purpose of experimentally determining receptive fields of individual neurons in the visual pathway. We modified the ALOPEX optimization in $[5,10,11]$ and introduced new versions in addition to the ones already known.

The ALOPEX process operates as follows:

- At each time step of the procedure, the variables that determine the cost function are changed simultaneously by small increments, and the cost function is re-evaluated.

- The changes in a variable depend stochastically on the change of the cost function and the change in that variable over the previous two time steps.

- Since the changes are cumulative, the value of each variable reflects at all times the dependence of the cost function to changes in that variable over all previous time steps.

- The process is guided by two free parameters, which are the size of the increments and the degree of stochasticity, i.e. the amplitude of noise.

For the purposes of the present simulation, the cost function $f$ of the system equates to the function $S_{\text {tip tilt }}$, defined by the integral shown in Eq. (3) and referring to the total light distribution measured on the masking area of the image. From Eqs. (3), (1), (2) and (4), it can be easily seen that the cost function of the system $S_{\text {tip tilt }}$ is a function of the tip and the tilt components of the mirror, $a_{1}$ and $a_{2}$. The restoration of the centroid of the speckled images is to be achieved by optimization of the cost function $S_{\text {tip tilt }}\left(a_{1}, a_{2}\right)$, via the appropriate changes in the slope of the mirror, i.e. via changes in the control variables $a_{1}$ and $a_{2}$. An optimization of $S_{\text {tip tilt }}\left(a_{1}, a_{2}\right)$ in real time will in turn improve the sharpness of the final, long exposure image.

Let $a_{i}^{(n)}$ be the value of the $i$ th control variable after the $n$th time step and let $S^{(n)}\left(a_{1}^{(n)}, a_{2}^{(n)}\right)$ be the value of the cost function. According to the version of the ALOPEX stochastic optimization algorithm presented in $[1,11]$, given the (random) initial conditions $a_{i}^{(0)}$, $a_{i}^{(1)}$ and $a_{i}^{(2)}(i=1,2)$, the value of the $i$ th control variable is evaluated, at each time step, according to the rule:

$$
a_{i}^{(n+1)}=a_{i}^{(n)}+c \Delta a_{i}^{(n)} \frac{\Delta S^{(n)}}{\left|\Delta S^{(n-1)}\right|}+g_{i}^{(n)},
$$$$
i=1,2, n \geqslant 3 \text {, }
$$

where $\Delta a_{i}^{(n)}=a_{i}^{(n)}-a_{i}^{(n-1)}$ and $\Delta S^{(n)}=S^{(n)}-$ $S^{(n-1)}$. The noise terms $g_{i}$ are essential ingredients in the process, as they provide the agitation necessary to drive the process [1,5,8-11]. The dynamics of the process depends strongly on the mean amplitude of the $g_{i}$ terms.

With use of the above version of the ALOPEX stochastic optimization algorithm, the value of parameter $c$ remains constant during the optimization process. For the results presented in the present paper, there is $c=0.4$.

For a more detailed discussion on the version of ALOPEX stochastic optimization algorithm presented by Eq. (11) see $[1,11]$. 
Characteristics of the method, such as

- effectiveness and speed of convergence in real time,

- no required knowledge of the dynamics of the system or of the functional dependence of the cost function on the control variables,

- easy and cost effective implementation

are the main factors that may lead to a successful implementation of the suggested method to real astronomical systems.

\section{Numerical experimentations}

\subsection{Definitions}

We define the following parameters:

- Parameter $\mu$, $\mu=\frac{\text { masking area diameter }}{\text { airy disk diameter }}$

investigates the performance of the method, as it pertains to the diameter of the masking area. Recall that the airy disk is the central disk of the irradiance distribution of the image of the point source (see Fig. 2).

- Parameter $\mu_{1}$,

$\mu_{1} \equiv \frac{D_{m}^{\text {restored }}}{D_{m}^{\text {distorted }}}$

estimates the restoration of the light intensity spread around the central maximum. $D_{m}^{\text {restored }}$ is the diameter of the light distribution at half the intensity maximum of the restored long exposure image (note that this parameter can be found in the bibliography as the FWHM (Full Width Half Maximum). $D_{m}^{\text {distorted }}$ is the diameter of the light distribution at half the intensity maximum of the distorted long exposure image.

- Parameter $\mu_{2}$,

$\mu_{2} \equiv \frac{S R_{\text {restored image }}}{S R_{\text {distorted image }}}$

compares the $S R$ values of the restored long exposure images to that of the distorted long exposure image.
A combination of parameters $\mu_{1}$ and $\mu_{2}$ is believed to give a sufficient measure of the restoration of the image sharpness.

\subsection{The restoration process}

The restoration process is assumed to start with the tip-tilt mirror aligned parallel to the image and pupil plane (i.e. for $\left.n=1, \phi_{c}^{(1)}(\rho, \theta)=0\right)$. The $n$th time step (where $n \geqslant 3$, as in Eq. (11)) starts with the calculation of the distorting phase $\phi^{(n)}(\rho, \theta)$ (i.e. the $n$th turbulent phase screen, see Section 2.2). As described by Eq. (2), the evaluation of the function $\delta^{(n)}(\rho, \theta)$ involves the subtraction of the correcting phase $\phi_{c}^{(n-1)}(\rho, \theta)$, which corresponds to the mirror's position at the previous time step $n-1$, from the distorting phase $\phi^{(n)}(\rho, \theta)$. The total light intensity on the masking area is then measured and the cost function $S_{\text {tip tilt }}^{(n)}$ is evaluated (see Eq. (3)). This value of the cost function is given as feedback for ALOPEX, see Eq. (11), for the calculation of the new position of the mirror (as described in the previous sections, ALOPEX calculates the coefficients $a_{1}^{(n)}$ and $a_{2}^{(n)}$ by which the correcting phase $\phi_{c}^{(n)}(\rho, \theta)$ is evaluated). We recall (see Eq. (11)) that ALOPEX optimization requires information from the two previous time steps, i.e. the values of $S_{\text {tip tilt }}^{(n-1)}, S_{\text {tip tilt }}^{(n-2)}, a_{1}^{(n-1)}$ and $a_{2}^{(n-1)}$. Fig. 5 illustrates the optimization process for the three successive time steps $n-2, n-1$ and $n(n \geqslant 3)$.

The success of the method is demonstrated through Fig. 6, which shows random samples of speckles, taken on the image plane as the exposure time progressed. The images shown in (a) are the result of the distorting atmosphere (ALOPEX optimization turned off), while the images in (b) are the result of a replay of the same time sequence of the turbulent phase screens of (a) (i.e they are subjected to the same distortion), but with correction by help the tip-tilt mirror (ALOPEX optimization turned on). We note that the three pairs of images shown in Fig. 6 correspond to the short exposure images of time steps $n=50$, $n=500$ and $n=1000$ of a 1000 -step optimization process. For these images, parameter $\mu$ was kept constant and equal to $\mu=1.875$ (see section below) and the severity of turbulence was $D / r_{0}=8.6$ (compare with Fig. 3). By simple observation of the images of Fig. 6, one may verify that, although turbulence tends 


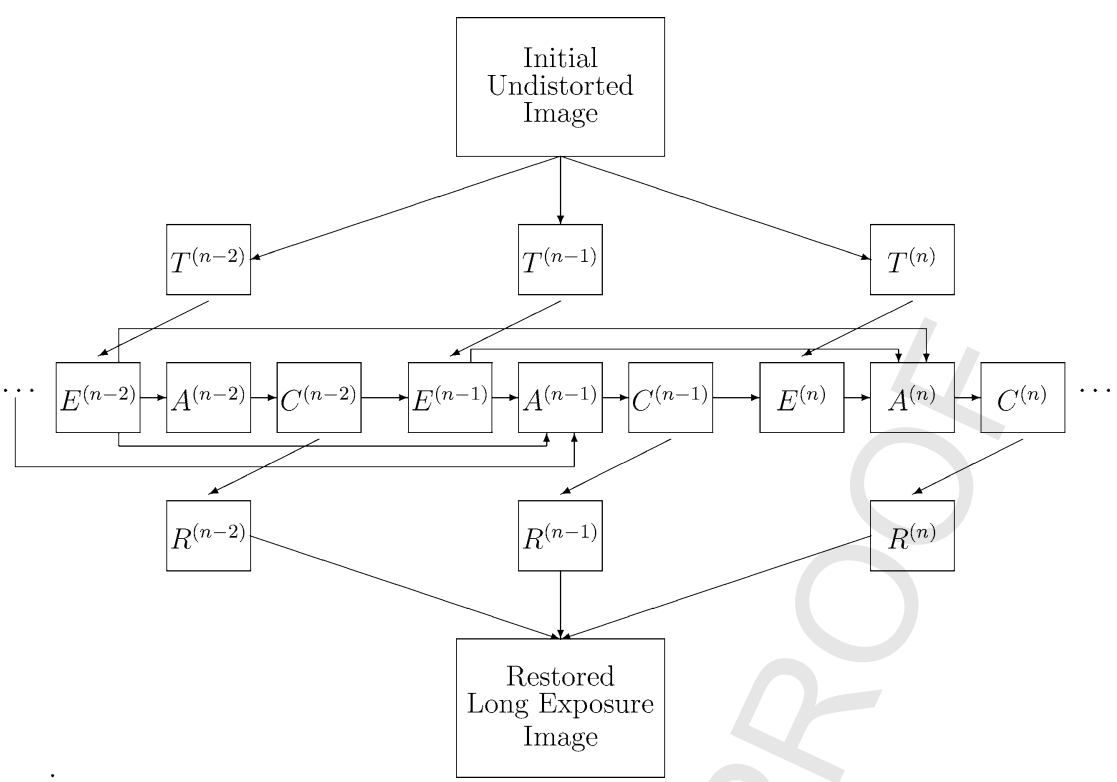

Fig. 5. Illustration of the optimization process for the three successive time steps $n-2, n-1$ and $n$. $T^{(n)}$ : Turbulent wavefront generator $E^{(n)}$ : Evaluation of the light intensity on the masking area. $A^{(n)}$ : ALOPEX stochastic optimization. $C^{(n)}$ : Wavefront Corrector (Tip-tilt mirror). $R^{(n)}$ : Restored position of the centroid of the speckles.

Table 1

Intensity of the speckled images

\begin{tabular}{|c|c|c|c|}
\hline & $\begin{array}{c}\text { Peak intensity } \\
\text { (distorted image) }\end{array}$ & $\begin{array}{c}\text { Central intensity } \\
I(0) \\
\text { (distorted image) }\end{array}$ & $\begin{array}{c}\text { Central intensity } \\
I(0) \\
\text { (restored image) }\end{array}$ \\
\hline 0 & 0.098 & 0.024 & 0.097 \\
\hline 1 & 0.018 & 0.009 & 0.016 \\
\hline 2 & 0.079 & 0.035 & 0.068 \\
\hline 3 & 0.136 & 0.125 & 0.132 \\
\hline 4 & 0.064 & 0.047 & 0.058 \\
\hline 5 & 0.078 & 0.009 & 0.064 \\
\hline 6 & 0.077 & 0.016 & 0.059 \\
\hline 7 & 0.147 & 0.065 & 0.105 \\
\hline 8 & 0.138 & 0.024 & 0.123 \\
\hline 9 & 0.054 & 0.036 & 0.050 \\
\hline 10 & 0.056 & 0.082 & 0.048 \\
\hline $\begin{array}{c}S R \\
\text { (superposition) }\end{array}$ & $I(0)_{\max }=0.945$ & 0.473 & 0.820 \\
\hline
\end{tabular}

to move the centroid of the speckles outside the masking area (Fig. 6(a)), the optimizing process manages to restore, in real time, the central position of the centroid and successfully calculate at each time step the tip and tilt parameters of the correcting mirror.

For a more detailed description of the process, we present the peak and central value of the intensity for ten speckle images. These images are random snapshots taken during a typical 1000-step exposure time, as previously, with and without the help of ALOPEX optimization. The results are presented in Table 1 (Fig. 6 includes three out of these ten images). The last two entries of Table 1 give the values of the central light intensity which correspond to 
1 (a)
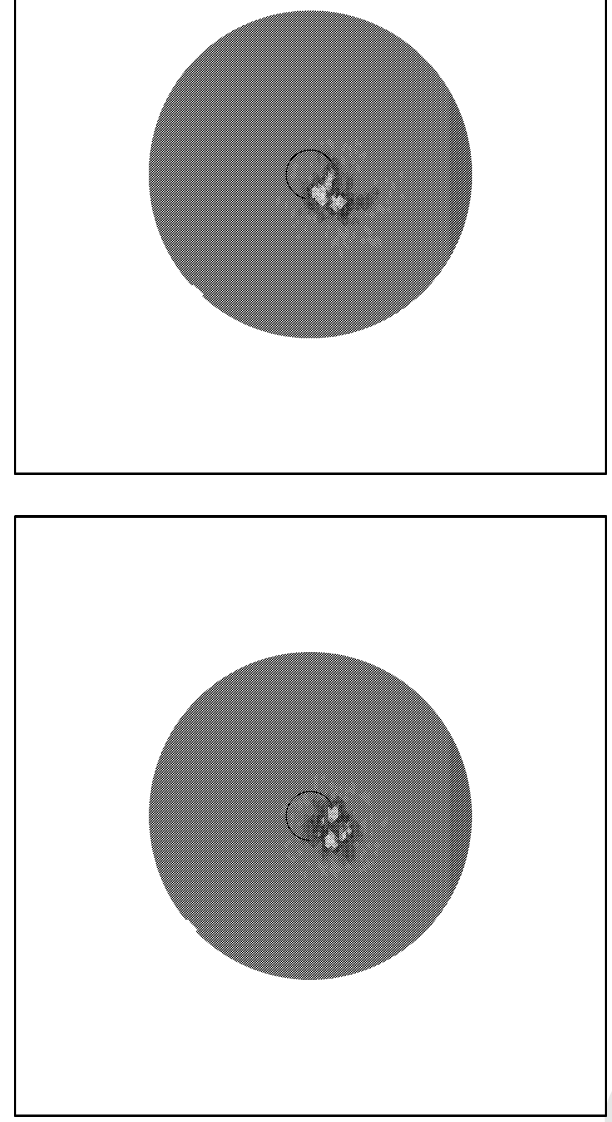

(a)

(a)

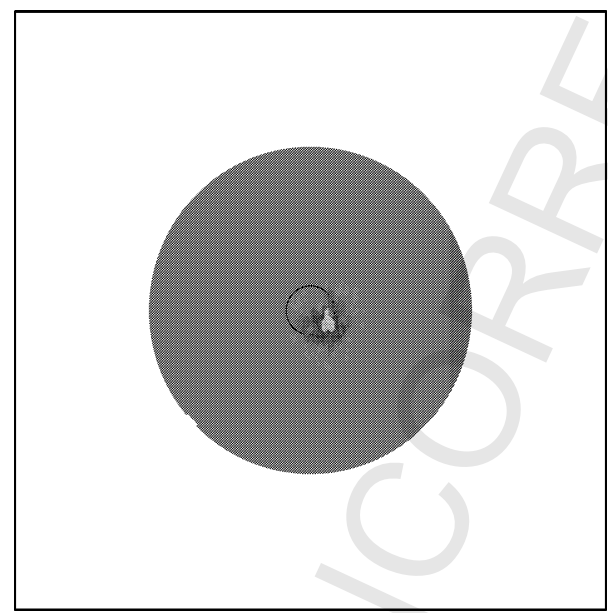

(b)

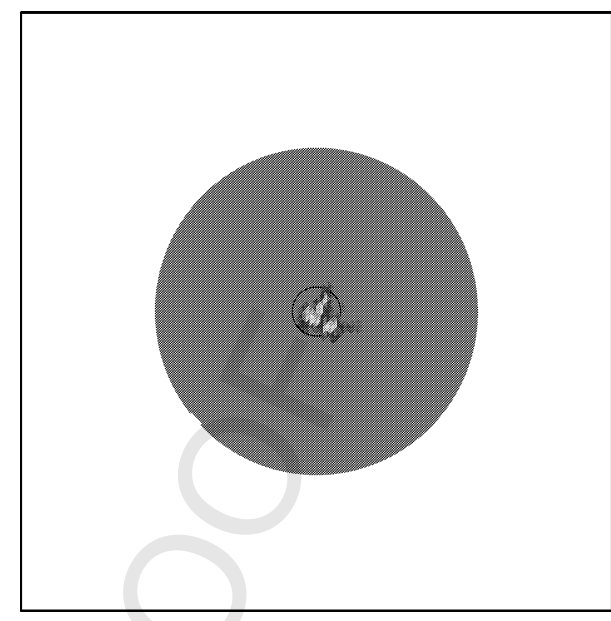

(b)

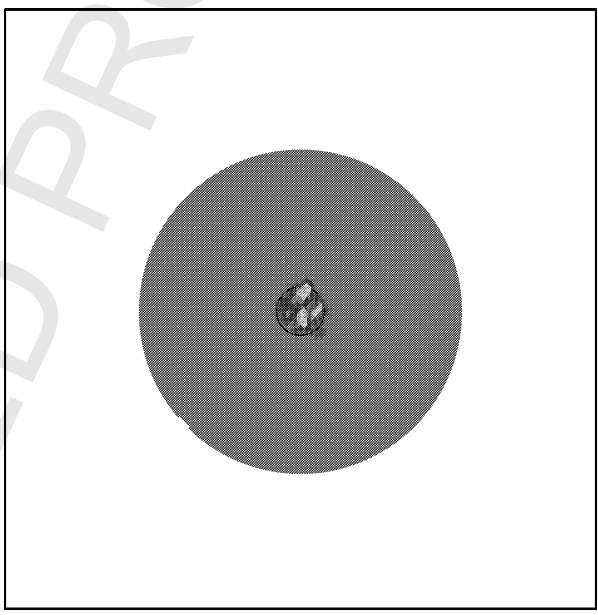

(b)

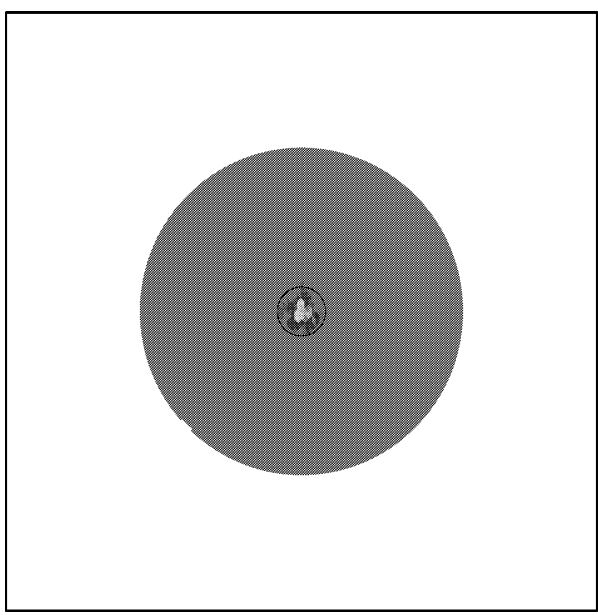

49

50

51

52

53

54

55

56

57

58

59

60

61

62

63

64

65

66

67

68

69

70

71

72

73

74

75

76

77

78

79

80

81

82

83

84

85

86

87

88

89

90

91

92

93

94

95

Fig. 6. Restoration of the centroid
a 1000-step optimization process. 
the superposition of the ten speckled images under study. These values were calculated to be $I(0)=$ 0.473 without and $I(0)=0.820$ with use of the optimizing method. For comparison, we evaluate the maximum possible value of the central irradiance distribution for the superposition of these ten speckle patterns, $I_{\max }(0)=0.945$. One can observe that the sharpness of the superposition of the ten speckled images under study is restored to $86.77 \%$ of its maximum value.

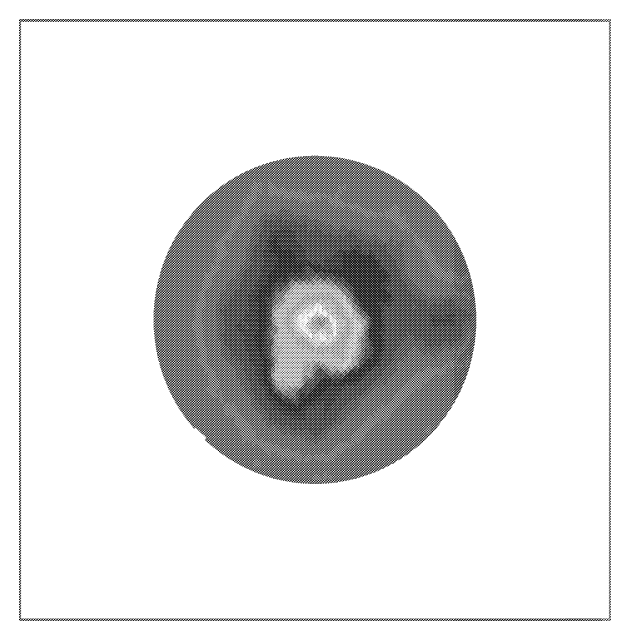

(a)

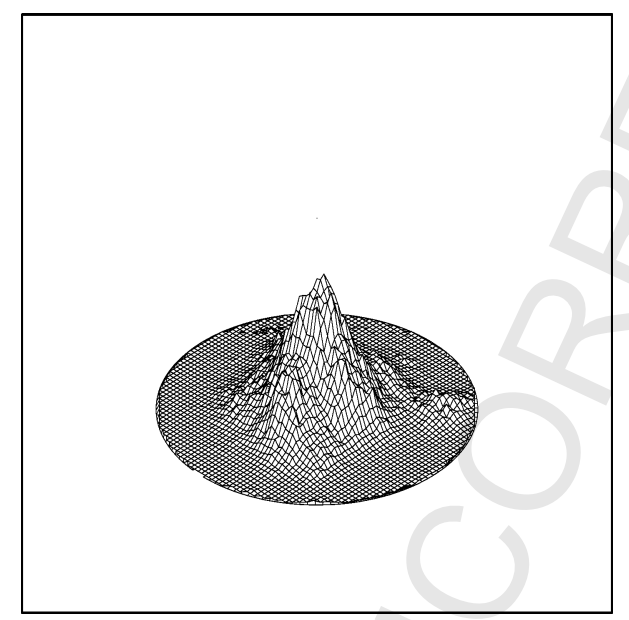

(b)

Fig. 7. A distorted image (a) as shown on the image plane and (b) irradiance distribution.
It is worth adding that, for the results presented in this paragraph, the mean value of the noise amplitude for the ALOPEX algorithm was kept equal to

$\left\langle g_{i}\right\rangle=1.964 \cdot 10^{-2} D_{\text {speckles }}$,

where $D_{\text {speckles }}$ is the average diameter of the speckle pattern.

This value of $\left\langle g_{i}\right\rangle$, as well as all the values of $\left\langle g_{i}\right\rangle$ 's that are presented in this study are the result of trial and error during the numerical simulation.

\subsection{The diameter of the masking area}

A representative long exposure image of the point source under observation typically looks like Fig. 7, which corresponds to a superposition of 1000 speckled images, like the ones shown in Fig. 6. To measure the restoration of the image sharpness, we compare the sharpness of Fig. 7, to the sharpness of the sample restored images shown in Fig. 8. These images, as well as Fig. 7, were obtained by the superposition of 1000 speckles.

Table 2 presents the dependence of the final sharpness of the image on the size of the masking area, through the values of parameters $\mu, \mu_{1}$ and $\mu_{2}$. Observe that the optimal image sharpness is derived by a masking area of diameter approximately twice as that of the airy disk $(\mu \approx 2)$. Figs. 9 and 10 illustrate the data of Table 2.

Table 2

Image restoration parameters with relation to the diameter of the masking area

\begin{tabular}{ccc}
\hline$\mu$ & $\mu_{1}$ & $\mu_{2}$ \\
\hline 0.25 & 0.563 & 1.209 \\
0.50 & 0.538 & 1.323 \\
0.75 & 0.531 & 1.469 \\
1.00 & 0.529 & 1.653 \\
1.25 & 0.523 & 1.763 \\
1.50 & 0.513 & 1.836 \\
1.75 & 0.500 & 1.928 \\
2.00 & 0.499 & 2.057 \\
2.25 & 0.501 & 1.892 \\
2.50 & 0.514 & 1.765 \\
2.75 & 0.526 & 1.692 \\
3.00 & 0.517 & 1.638 \\
3.75 & 0.528 & 1.609 \\
5.00 & 0.539 & 1.551 \\
6.25 & 0.543 & 1.364 \\
7.50 & 0.548 & 1.267 \\
\hline
\end{tabular}


(a)

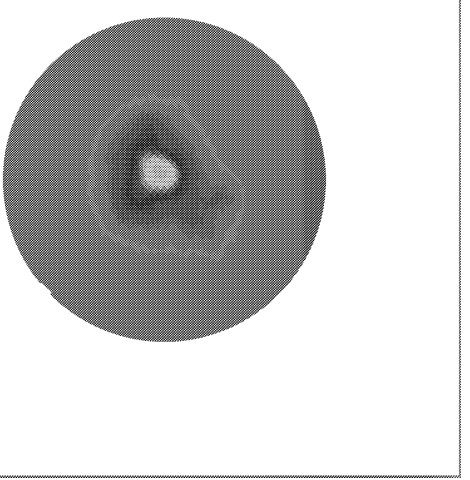

(a)

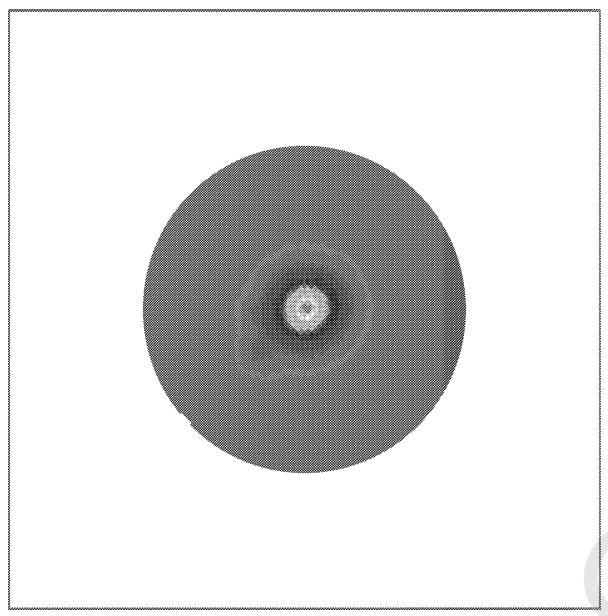

(a)

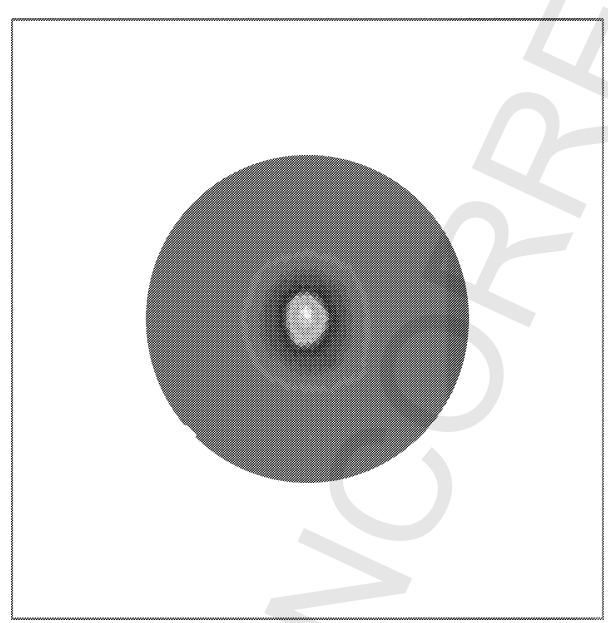

(b)

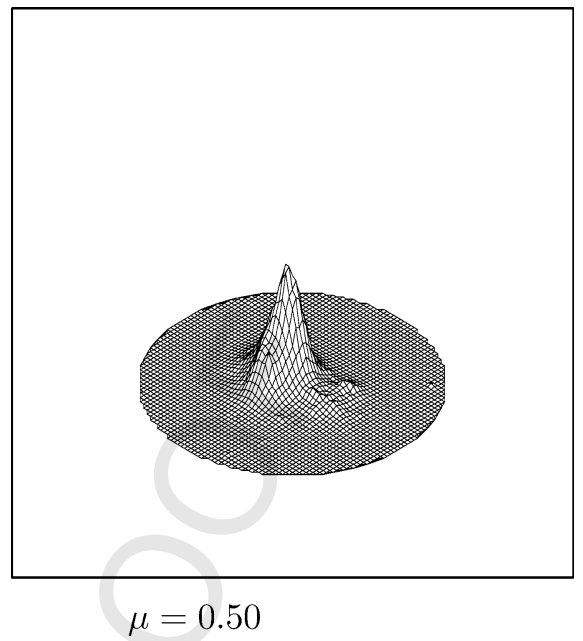

(b)

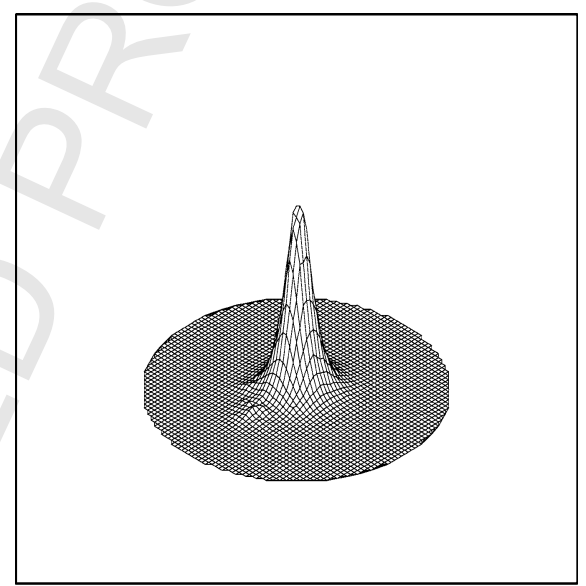

$\mu=2.00$

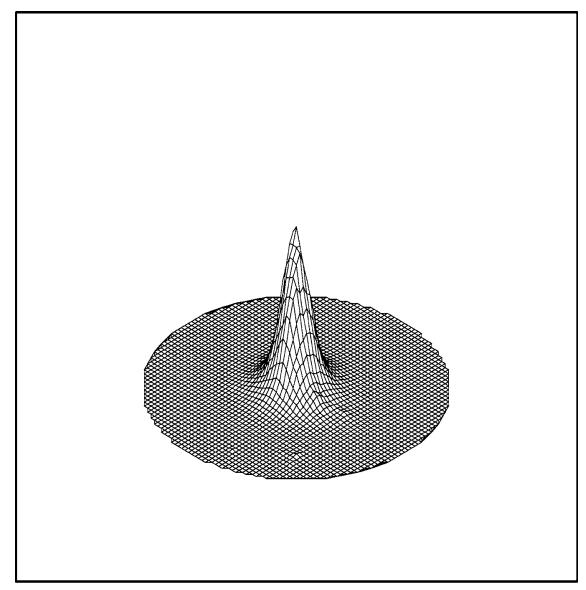

(b)
49

50

51

52

53

54

55

56

57

58

59

60

61

62

63

64

65

66

67

68

69

70

71

72

73

74

75

76

77

78

79

80

81

82

83

84

85

86

87

88

89

90

91

92

93

94

95

96

Fig. 8. Samples of restored images for different values of $\mu$. (a) As shown on the image plane, (b) irradiance distribution. 


\subsection{Image restoration versus the mean velocity of speckles}

The mean velocity of the speckle pattern is directly related to the rate of change of the turbulent at-

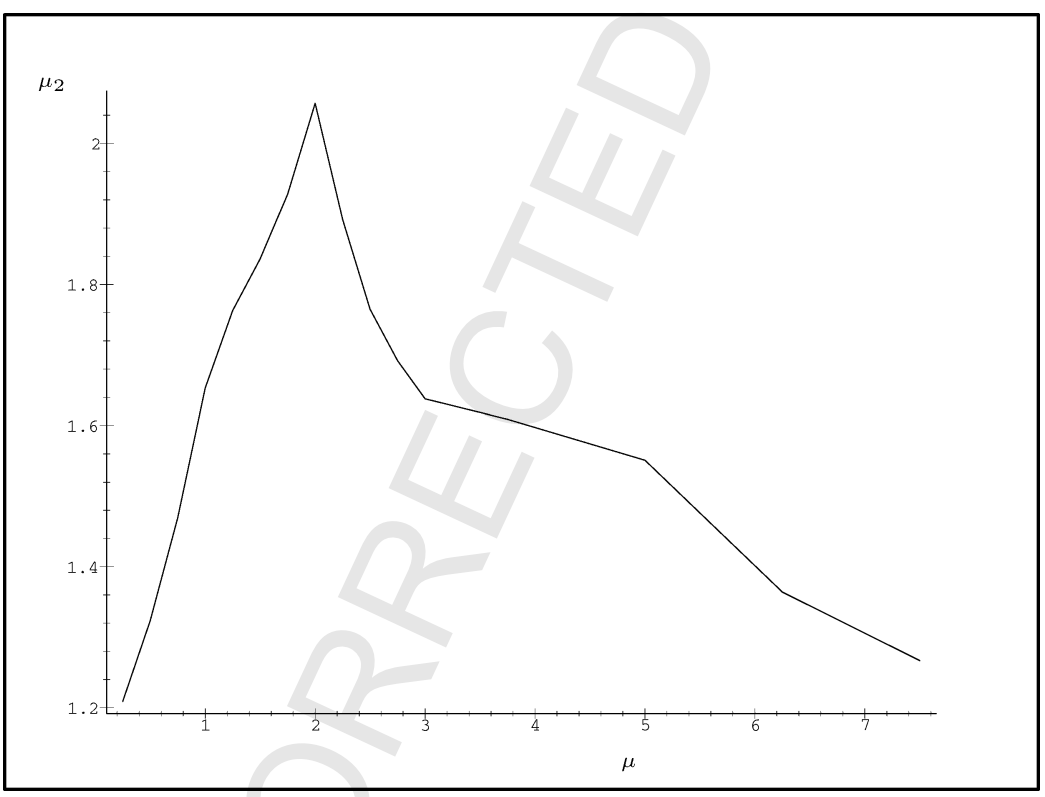

Fig. 10. SR restoration versus the diameter of the masking area. 


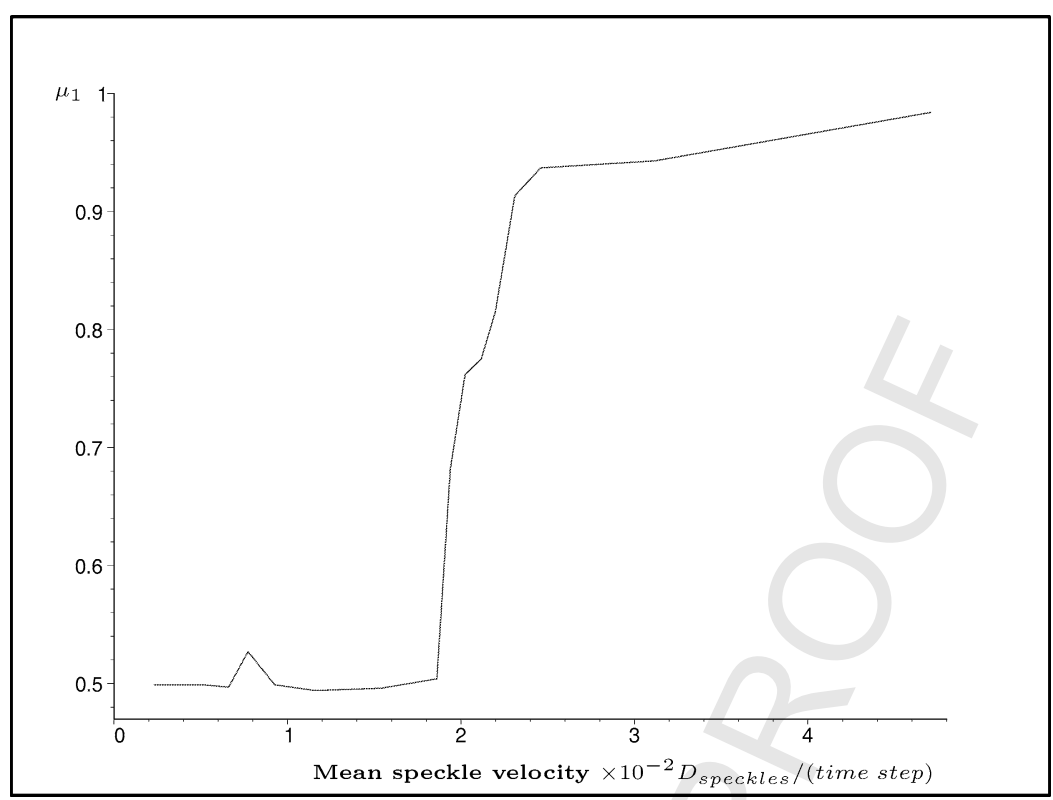

Fig. 11. Intensity spread restoration versus mean speckle velocity.

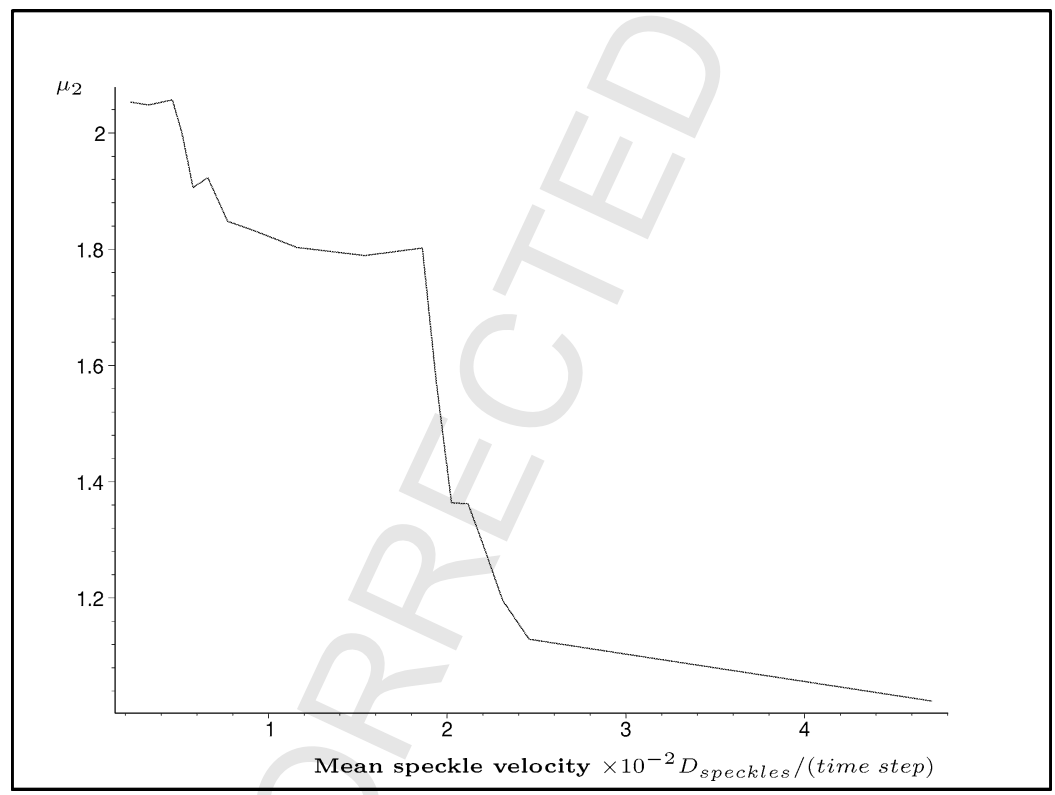

Fig. 12. SR restoration versus mean speckle velocity.

of change of the position of the centroid of the speckles. In contrast to previous work, which considers velocities on the pupil plane, we define a quantity which can be rigorously measured on the image plane, as follows: Assuming short time intervals $\Delta t$ of the order of a few time steps, corresponding to times shorter than the correlation time of the turbulence, we can calculate a mean value of the rate of change of the position of the centroid of the speckles. We define the quantity $\bar{v}_{\text {speckles }}$ to describe the rate of change of the position 
of the centroid of the speckles. This mean velocity of speckles, $\bar{v}_{\text {speckles}}$, may be expressed in terms of the average diameter of the speckle pattern, $D_{\text {speckles }}$, and in units of $D_{\text {speckles }} /$ (time step).

\subsubsection{Keeping the mean noise amplitude constant}

The image restoration process was studied for various values of $\bar{v}_{\text {speckles. }}$. For the results presented in

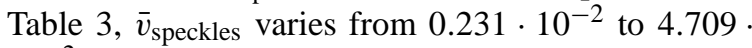

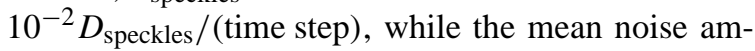
plitude is constant and equal to $\left\langle g_{i}\right\rangle=1.964$. $10^{-2} D_{\text {speckles. }}$

Table 3

Restoration parameters versus the mean image velocity

\begin{tabular}{ccc}
\hline $\bar{v}_{\text {speckles }}\left[D_{\text {speckles }} /\right.$ (time step) $]$ & $\mu_{1}$ & $\mu_{2}$ \\
\hline $4.709 \cdot 10^{-2}$ & 0.984 & 1.023 \\
$3.118 \cdot 10^{-2}$ & 0.943 & 1.098 \\
$2.458 \cdot 10^{-2}$ & 0.937 & 1.129 \\
$2.310 \cdot 10^{-2}$ & 0.913 & 1.196 \\
$2.198 \cdot 10^{-2}$ & 0.815 & 1.294 \\
$2.117 \cdot 10^{-2}$ & 0.775 & 1.362 \\
$2.024 \cdot 10^{-2}$ & 0.762 & 1.364 \\
$1.939 \cdot 10^{-2}$ & 0.682 & 1.571 \\
$1.861 \cdot 10^{-2}$ & 0.504 & 1.802 \\
$1.539 \cdot 10^{-2}$ & 0.496 & 1.789 \\
$1.159 \cdot 10^{-2}$ & 0.494 & 1.803 \\
$0.927 \cdot 10^{-2}$ & 0.499 & 1.831 \\
$0.772 \cdot 10^{-2}$ & 0.527 & 1.848 \\
$0.661 \cdot 10^{-2}$ & 0.497 & 1.923 \\
$0.578 \cdot 10^{-2}$ & 0.498 & 1.906 \\
$0.514 \cdot 10^{-2}$ & 0.499 & 2.002 \\
$0.462 \cdot 10^{-2}$ & 0.499 & 2.057 \\
$0.330 \cdot 10^{-2}$ & 0.499 & 2.048 \\
$0.231 \cdot 10^{-2}$ & 0.499 & 2.053 \\
\hline
\end{tabular}

Figs. 11 and 12 illustrate the results presented in Table 3.

One can observe that, for $\bar{v}_{\text {speckles }}<1.939 \cdot 10^{-2}$ $D_{\text {speckles }}$ (time step) the mean amplitude of noise $\left\langle g_{i}\right\rangle$ implemented can be kept optimally constant, even though its average numerical values are higher than the average values of the displacement of the centroid of the speckles at each time step. This can be explained due to the intrinsic properties of ALOPEX stochastic optimization, see Eq. (11). For velocities $\bar{v}_{\text {speckles }}>$ $1.939 \cdot 10^{-2} D_{\text {speckles }}$ (time step) the method fails to satisfactory restore the image. Note, however, that such velocities correspond to fast rate of change of the position of the centroid of the speckles and hence represent worse than average weather conditions. We believe that in such cases of strong atmospheric distortion, the problem of satisfactory image sharpness restoration will be overcome by appropriately changing the mean amplitude $\left\langle g_{i}\right\rangle$ of the noise. The section that follows justifies our hypothesis.

\subsubsection{Changing the mean noise amplitude}

We investigate the effect of the implementation of increasing mean noise amplitudes $\left\langle g_{i}\right\rangle$ in the case where the mean image velocity $\bar{v}_{\text {speckles }}$ exceeds the limit of $1.939 \cdot 10^{-2} D_{\text {speckles }} /($ time step). As shown in Table 4, which summarizes the results of the simulation, there is an optimal mean noise amplitude $\left\langle g_{i}\right\rangle$ for each $\bar{v}_{\text {speckles}}$, resulting in a satisfactory restoration of the image sharpness.

In Figs. 13 and 14, which illustrate the data of Table 4 , the results of implementing a mean noise amplitude $\left\langle g_{i}\right\rangle$, which increases as $\bar{v}_{\text {speckles }}$ increases, are presented with solid lines. Dashed lines correspond

Table 4 


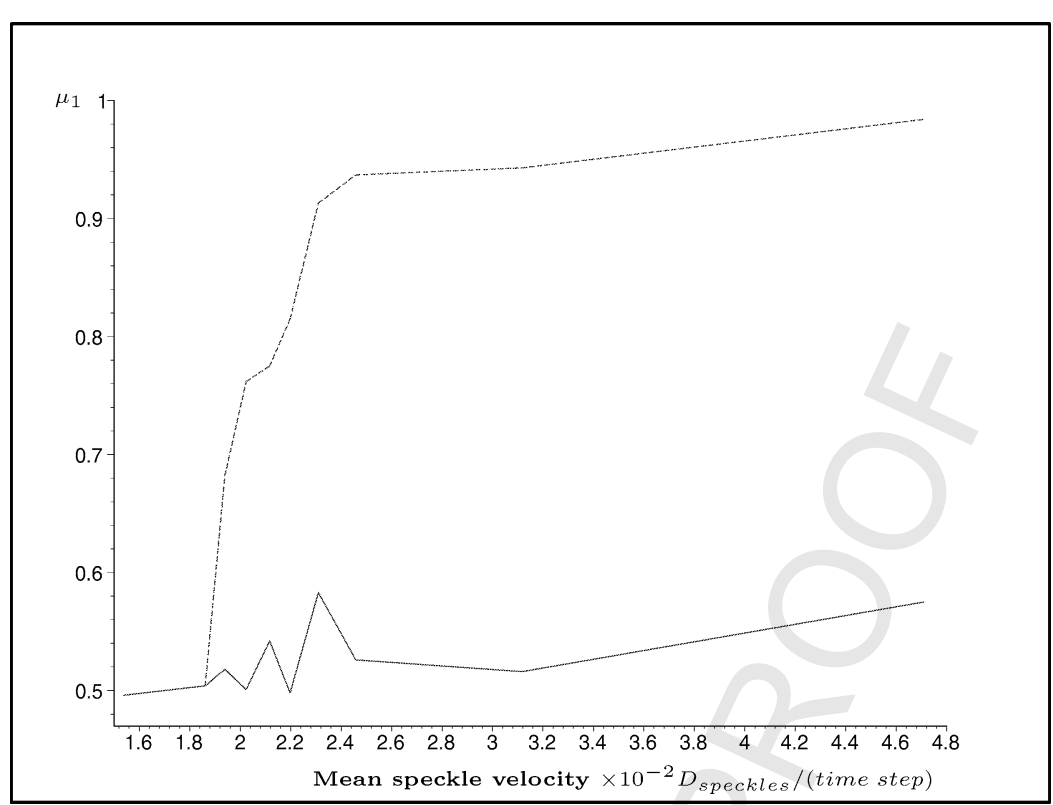

Fig. 13. Intensity spread restoration versus mean speckle velocity, in relation to the noise amplitude: $\cdots$ keeping $\left\langle g_{i}\right\rangle$ 's constant, - changing the $\left\langle g_{i}\right\rangle$ 's.

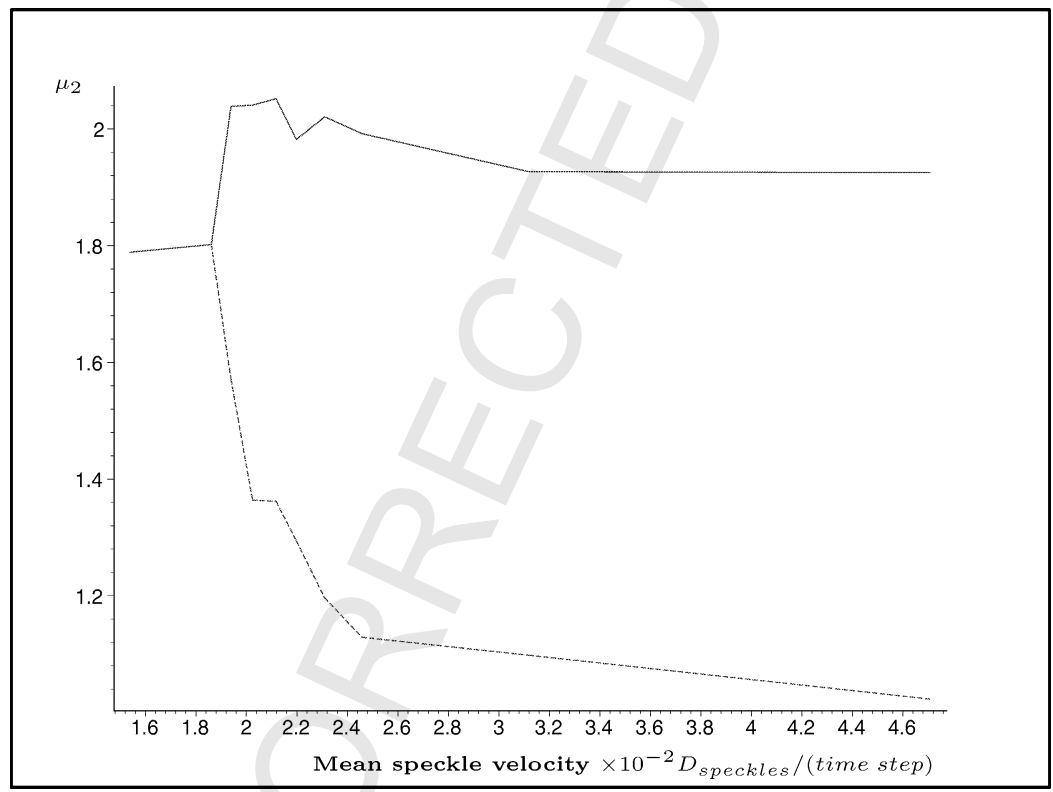

Fig. 14. SR restoration versus mean speckle velocity, in relation to the noise amplitude: $\cdots$ keeping $\left\langle g_{i}\right\rangle$ 's constant, - changing the $\left\langle g_{i}\right\rangle$ 's.

to the data of Figs. 11 and 12 (Table 3). The success of the implementation is apparent.

Fig. 15 shows the necessary increase in the noise amplitude when the mean image velocities $\bar{v}_{\text {speckles }}$ be- come larger than $1.939 \cdot 10^{-2} D_{\text {speckles }} /($ time step), i.e. under weather conditions which are worse than average. As can be observed, the mean noise amplitude can be assumed to be linearly depended on the mean veloc- 
ity of the speckles on the image plane, $\bar{v}_{\text {speckles. }}$ More specifically, a linear regression of the mean noise amplitude $\left\langle g_{i}\right\rangle$ on the mean velocity of speckles, $\bar{v}_{\text {speckles }}$, can be calculated:

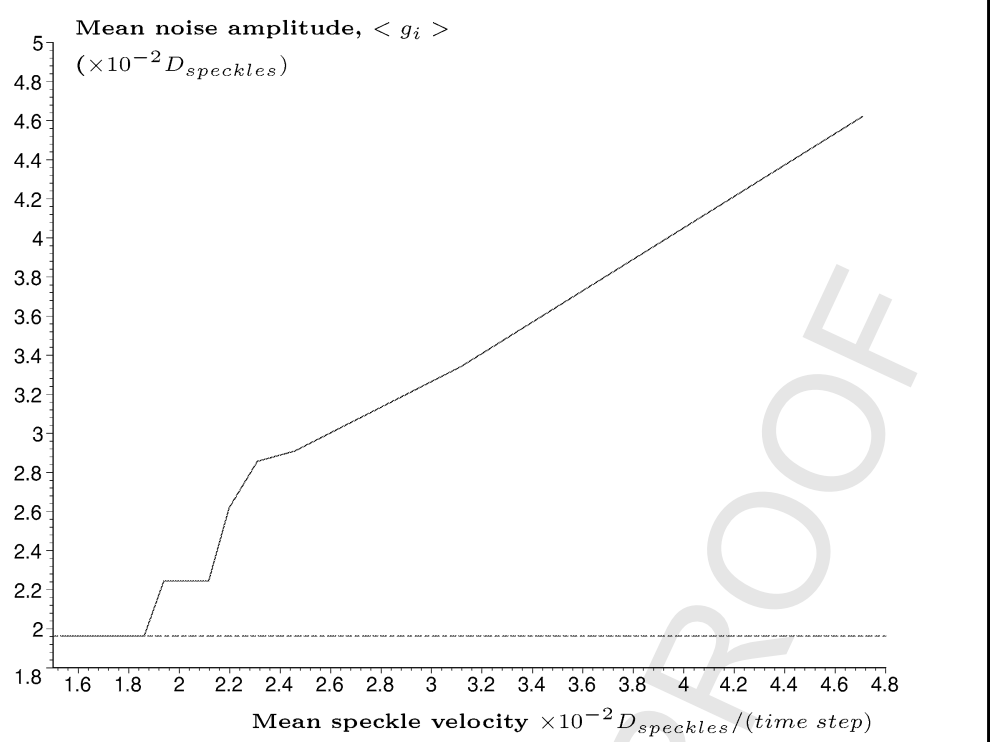

Fig. 15. Optimal noise amplitude versus speckle velocity: $\cdots$ keeping $\left\langle g_{i}\right\rangle$ 's constant, — changing the $\left\langle g_{i}\right\rangle^{\prime}$ 's.

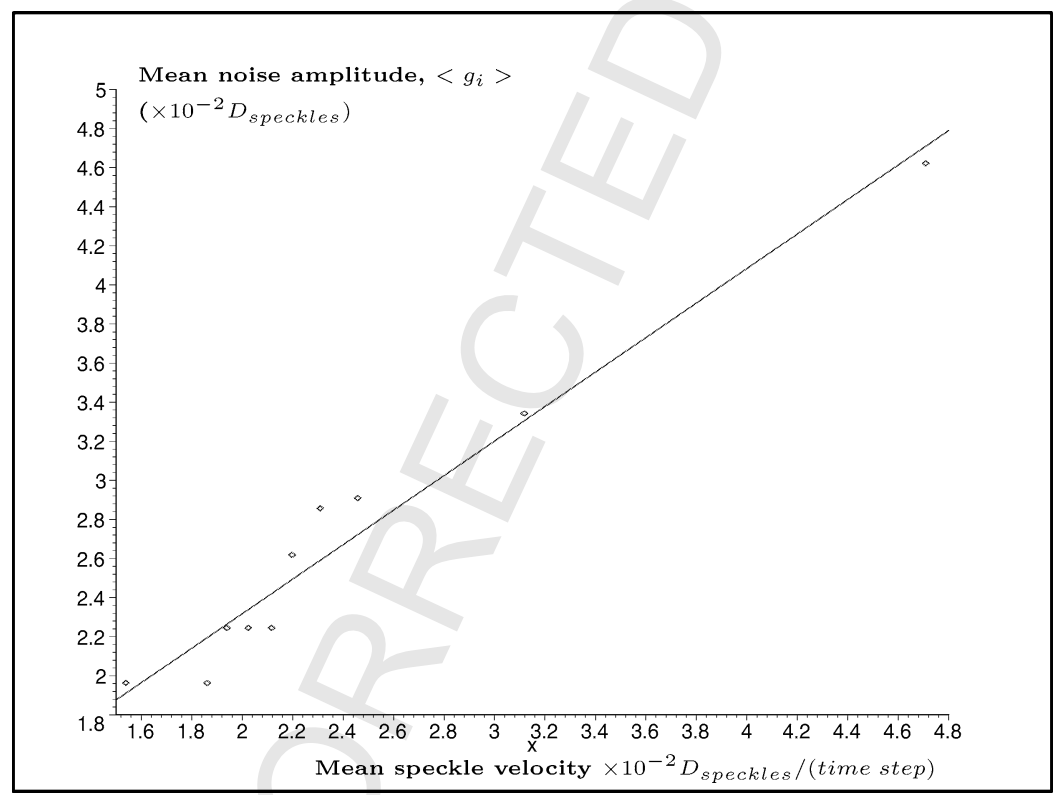

Fig. 16. Linear regression of optimal noise amplitude on the mean speckle velocity. 
mula is $r=0.97849$. Fig. 16 shows the above linear regression.

\section{Conclusions}

The present work investigates the optimization of an image of a point star using a computer simulated tip-tilt adaptive optics system. The optimization of the long exposure images is achieved by use of a stochastic optimization algorithm. The tip-tilt mirror is rotated in such a way that the position of the centroid of the short exposure images is restored, in real time, inside the central area of the image plane (the masking area). The optimal diameter of the masking area is found to be approximately twice the airy disk of the point star. We also found that for optimal image restoration and under conditions of good or medium seeing, the mean amplitude of the required noise in the algorithm can be kept constant during the optimization process. For worse than average weather conditions, the optimal mean noise amplitude and the mean velocity of the short exposure images are found to be linearly depended. By use of the optimization method presented here, the reduction of the distorting effects of the atmosphere are achieved in real time in a way which is far simpler, inexpensive and easy to implement than the currently used methods. Our optimizing system, being able to provide images of high quality and sharpness for all ground-based telescopes, is a highly competitive application to a very complicated and demanding problem.

\section{References}

[1] M.S. Zakynthinaki, Stochastic optimization for adaptive correction of atmospheric distortion in astronomical observation, PhD thesis, Technical University of Crete, 2001.

[2] H.W. Babcock, PASP 65 (1953) 229.

[3] H.W. Babcock, J. Optical Soc. Amer. 48 (1958) 500.

[4] R.J. Noll, J. Optical Soc. Amer. 66 (1976).

[5] Y.G. Saridakis, M.S. Zakynthinaki, T.E. Kalogeropoulos, Internat. J. Appl. Sci. Comput. 5 (3) (1999) 252.

[6] A.N. Kolmogorov, in: S.K. Friedlander, L. Topper (Eds.), Turbulence, Interscience, New York, 1965.

[7] R.G. Lane, A. Glindemann, J.C. Dainty, Waves in Random Media 2 (1992) 209.

[8] E. Harth, E. Tzanakou, Vision Res. 14 (1974) 1475.

[9] T. Tzanakou, R. Michalak, E. Harth, Biol. Cybernet. 35 (1979) 161.

[10] T.E. Kalogeropoulos, Y.G. Saridakis, M.S. Zakynthinaki, Comput. Phys. Comm. 99 (1997) 255.

[11] Y.G. Saridakis, M.S. Zakynthinaki, in: Proc. of the 3rd Hellenic-European Conference on Mathematics and Informatics, LEA, Athens, 1996, p. 251.

[12] R.K. Tyson, Principles of Adaptive Optics, Academic Press, 1991.

[13] D.L. Fried, J. Optical Soc. Amer. 55 (1965). 